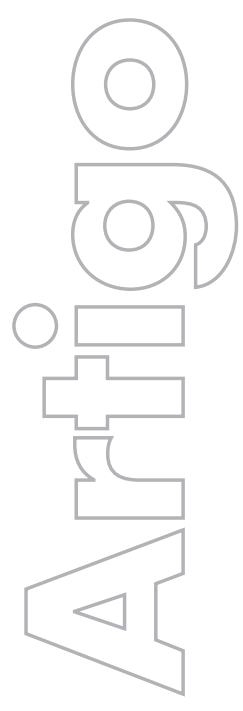

Geografia da mortalidade infantil do Brasil: variações espaciais e desigualdades territoriais

Rivaldo Faria

UFSM

\section{revista}

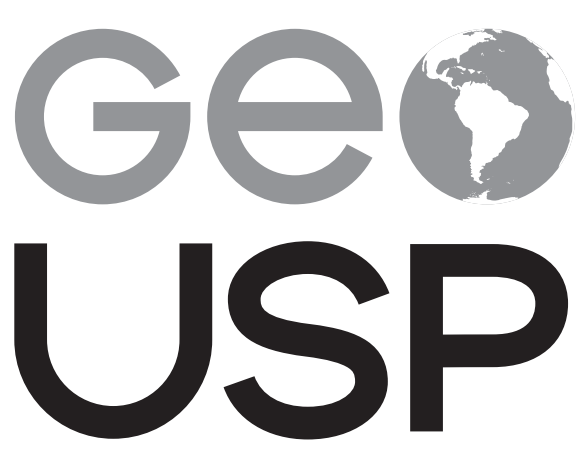

espaço e tempo

Volume $20 \cdot n^{\circ} 3(2016)$
Como citar este artigo:

FARIA, R. Geografia da mortalidade infantil do Brasil: variações espaciais e desigualdades territoriaissx. Geousp - Espaço e Tempo (Online), v. 20, n. 3, p. 602-618, mês. 2016. ISSN 2179-0892.

p. $602-618$

Disponível em: <http://www.revistas.usp.br/geousp/issue/ view/6465>. doi: http://dx.doi.org/10.11606/issn.2179-0892. geousp.2014.84539.

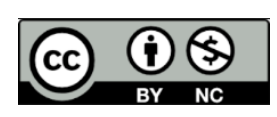

Este artigo está licenciado sob a Creative Commons Attribution 4.0 License. 


\title{
Geografia da mortalidade infantil do Brasil: variações espaciais e desigualdades territoriaisl
}

\section{Resumo}

Este artigo discute as variações espaciais e as desigualdades territoriais em função dos indicadores de mortalidade infantil do Brasil. $\bigcirc$ estudo foi feito com todos os óbitos infantis e nascidos vivos registrados em sistemas oficiais nos anos de 2012 e 2013 nas 558 microrregiões brasileiras. Os indicadores foram calculados e estimados pelo método bayesiano empírico, e as características do território foram analisadas por meio de um indicador composto chamado índice de privação social (IPS). Os resultados indicaram altos coeficientes de mortalidade infantil (CMI) nas microrregiões dos estados do Nordeste e do Norte do país e fatores associados relativos à idade da mãe, a doenças infecciosas, a vazios assistenciais e à falta de acompanhamento pré-natal. Nas microrregiões dos estados do Sudeste e do Sul e de parte do Centro-Oeste, os CMI são menos elevados, e os fatores associados estão mais relacionados com a prematuridade e o baixo peso no nascimento.

Palavras-chave: Mortalidade infantil. Desigualdades territoriais. Privação social. Prematuridade. Baixo peso.

\section{Geography of infant mortality in Brazil: spatial variations and inequalities territorial}

\begin{abstract}
The aim of this article is to analyze the spatial variations of the infant mortality indicators in Brazil. The study was done with all live births and infant deaths collected in official system in the years of 2012 and 2013 in 558 Brazilian microregions. The indicator was estimated and calculated by empirical bayesian method. The characteristics of territory were analyzed using a composed index called Index of Social Deprivation (ISD). The results showed high infant mortality coefficient (IMC) in microregions of northeast and north states and reasons associated to mother age, infectious diseases,
\end{abstract}

Trabalho financiado pelo Conselho Nacional de Desenvolvimento Científico e Tecnológico (CNPq). 
empty healthcare and lack of prenatal care. In the southeastern, Southern and Midwest, the IMC are lower and the more important associate factors are prematurity and low birth weight.

Keywords: Infant mortality. Inequalities territorial. Social deprivation. Prematurity. Birth weight.

\section{Introdução}

A mortalidade infantil é um dos indicadores de saúde mais sensíveis às condições sociais relativas ao território (Costa et al., 2001; Goldani et al., 2001; Szwarcwald; Andrade; Bastos, 2002). Por isso, talvez nenhum outro indicador expresse mais claramente a expressão miltoniana segunda a qual "cada homem vale pelo lugar onde está" (Santos, 1987, p. 107). Com recurso à paráfrase, cada criança vive menos ou mais ou simplesmente sobrevive, dependendo do ponto do território onde está (Norman et al., 2008). É assim no Brasil, no mundo ou numa cidade.

A mortalidade infantil, como a fome, é um problema do território (Faria; Santana, 2015; Carvalho et al., 2015). Por isso, novamente como a fome, é um indicador social e de saúde de extrema gravidade. Na verdade, nenhuma criança deveria morrer no seu primeiro ano de vida (Nolte; Mckee, 2004). Além disso, diferentemente de outros grupos sociais como as mulheres, os idosos, adolescentes e jovens, a criança (nesse caso, com menos de um ano de vida) é totalmente dependente das condições de segurança (alimentar, domiciliar, vacinal, entre outras) que the são oferecidas. Antes mesmo do nascimento, a sobrevida do bebê depende diretamente das condições da gestação e do parto (Victora; Barros, 2001), cujas implicações serão sentidas ao longo de toda a sua vida adulta. Por isso, talvez nenhum outro indicador de saúde seja tão estratégico para o desenvolvimento territorial, com enormes implicações, por exemplo, no famigerado índice de desenvolvimento humano (IDH).

Não é de estranhar que o estudo da mortalidade infantil tenha enorme conteúdo geográfico, seja como expressão do território, seja como determinante para sua compreensão (Campbell; Apparicio; Day, 2015; Faria; Santana, 2015). Invulgar é a pequena presença de geógrafos dedicados ao tema. No Brasil, os estudos de geografia da saúde ainda tendem fortemente a compreender as doenças infecciosas, sobretudo as vetoriais. ${ }^{2}$ Isso fez avançar a produção de uma geografia de superação dos estudos ecológicos ligados essencialmente às causalidades, como os de influência da tropicologia médica. Entretanto, será ainda necessário avançar na compreensão das doenças crônico-degenerativas e da situação de saúde de grupos específicos como os idosos, adolescentes, jovens, crianças e mães.

2 De acordo com Guimarães (2015, p. 11, grifo nosso), "as pesquisas dos geógrafos brasileiros estão concentradas em duas abordagens principais: o estudo dos padrões espaciais das doenças predominantemente infectocontagiosas (como dengue, malária, leishmaniose) e o estudo da oferta e demanda de geografia da saúde". 
A análise do indicador de mortalidade infantil ganha ainda uma relevância singular nesse ano de avaliação dos pactos celebrados no âmbito dos Objetivos de Desenvolvimento do Milênio (ODM) (WHO, 2005). Há uma ampla divulgação governamental do alcance antecipado da meta de redução de dois terços do coeficiente de mortalidade infantil no Brasil, comparativamente ao ano de 1990 (Ipea, 2014). Tarefa cumprida, mas que esconde realidades (municípios, microrregiões e até regiões inteiras) cujo coeficiente parece estar estacionado no século passado. Além disso, para efeito das novas metas e programas ainda em construção, uma análise realista pode também contribuir. Talvez seja adequado pensar não apenas o coeficiente em si, mas o coeficiente no território.

Este artigo, o primeiro de uma série de estudos que vimos desenvolvendo sobre a dinâmica geográfica da mortalidade infantil no Brasil, propõe analisar as variações espaciais e as desigualdades territoriais desse indicador em anos recentes (2012 e 2013), no nível das microrregiões (558) administrativas do IBGE. Além dos indicadores relativos aos nascidos vivos e ao óbitos infantis, foram ainda investigadas as características a eles associadas (prematuridade, baixo peso, idade da mãe, doenças infecciosas); a cobertura da atenção médica básica; o acesso ao pré-natal e as características sociais do território (analisadas a partir de um índice composto de privação social denominado índice de privação social).

texto foi organizado em três seções: a descrição metodológica, a apresentação dos resultados (subdividida, por sua vez, em três subseções: o coeficiente de mortalidade infantil, fatores associados como prematuridade, baixo peso, idade da mãe e doenças infecciosas e parasitárias, e a atenção e o acesso aos serviços de saúde e a privação social) e a discussão. E as considerações finais apresentam perspectivas para o debate.

\section{Material e métodos}

O estudo foi realizado com toda a população de nascidos vivos (5.809.816) e óbitos infantis (78.089) registrados nos anos 2012 e 2013 no nível das microrregiões (558) do Instituto Brasileiro de Geografia e Estatística (IBGE). $\bigcirc$ período de análise foi assim definido porque incorpora apenas os registros realizados nos novos formulários de declaração de nascidos vivos e óbitos infantis implantados pelo Ministério da Saúde no ano de 2011 (Brasil, $2012)^{3}$ e, ao mesmo tempo, permite, com dados agregados em dois anos, aumentar a população estudada e dar mais confiabilidade às taxas. Da mesma forma, no nível das microrregiões, a escala espacial permite agregar os dados de municípios muito pequenos e onde nem sempre ocorre óbito infantil, ${ }^{4}$ o que concorre para atenuar a generalização estatística na estimativa de grupos populacionais muito pequenos ou inexistentes (como é o caso da falta de óbito infantil em muitos municípios).

\footnotetext{
3 A alteração nos formulários de declaração de nascidos vivos e óbitos infantis em 2011 permitiu reduzir os sub-registros e aumentar a resolutividade em pelo menos $3 \%$.

4 Especificamente no período analisado (2012-2013), 659 municípios brasileiros não registraram óbito infantil.
} 


\section{(a) Levantamento dos dados e construção dos indicadores}

Todos os dados deste estudo foram coletados em fontes secundárias oficiais, nomeadamente no Sistema de Informação de Nascidos Vivos (Sinasc); Sistema de Informação de Mortalidade (SIM); Cadastro Nacional de Equipamentos de Saúde (Cnes) e no Banco de Dados Agregados (Sidra) do IBGE. O Quadro 1 apresenta, para cada um dos oito indicadores elaborados, conforme objetivos previamente definidos, os dados brutos efetivamente levantados.

\section{Quadro 1-Dados brutos levantados de cada indicador e sua(s) respectiva(s) fonte(s)}

\begin{tabular}{|c|c|c|}
\hline indicador & dados brutos utilizados & fonte(s) \\
\hline coeficiente de mortalidade infantil (TMI) & nascidos vivos e óbitos infantis & Sinasc-SIM \\
\hline $\begin{array}{l}\text { coeficiente de óbitos infantis relacionados a } \\
\text { prematuridade (Coip) }\end{array}$ & $\begin{array}{l}\text { óbitos infantis (total) e óbitos infantis } \\
\text { de nascença prematura }\end{array}$ & SIM \\
\hline $\begin{array}{l}\text { coeficiente de óbitos infantis relacionados a } \\
\text { baixo peso (Coibp) }\end{array}$ & $\begin{array}{l}\text { óbitos infantis (total) e óbitos infantis } \\
\text { de nascença de baixo peso(ii) }\end{array}$ & SIM \\
\hline $\begin{array}{l}\text { coeficiente de óbitos infantis relacionados a } \\
\text { algumas doenças infecciosas e parasitárias } \\
\text { (Coidip) }\end{array}$ & $\begin{array}{l}\text { óbitos infantis (total) e óbitos } \\
\text { infantis relativos a algumas doenças } \\
\text { infecciosas e parasitárias (iii) }\end{array}$ & SIM \\
\hline $\begin{array}{l}\text { coeficiente de óbitos infantis de mães adoles- } \\
\text { centes (Coima) }\end{array}$ & $\begin{array}{l}\text { óbitos infantis (total) e óbitos infantis } \\
\text { de mães entre } 10 \text { e } 19 \text { anos de idade }\end{array}$ & SIM \\
\hline $\begin{array}{l}\text { coeficiente de nascidos vivos que receberam } \\
\text { sete ou mais consultas pré-natais durante a } \\
\text { gestação (CNV } \geq 7 \mathrm{CPN})\end{array}$ & $\begin{array}{l}\text { nascidos vivos (total) e nascidos vivos } \\
\text { que fizeram sete ou mais consultas } \\
\text { pré-natais }\end{array}$ & Sinasc \\
\hline médicos do SUS por habitante (RMSUS) & $\begin{array}{l}\text { número de médicos por } \\
\text { especialidades }{ }^{(i v)} \text { selecionadas e popu- }^{\text {lação absoluta residente }}\end{array}$ & $\begin{array}{l}\text { Cnes e } \\
\text { Sidra }\end{array}$ \\
\hline índice de privação social (IPS) & $\begin{array}{l}\text { mulheres em idade reprodutiva (to- } \\
\text { tal); mulheres analfabetas em idade } \\
\text { reprodutiva; domicilios particulares } \\
\text { permanentes (total); domicílios parti- } \\
\text { culares permanentes sem instalação } \\
\text { sanitária; domicílios com renda de até } \\
\text { meio salário-mínimo }\end{array}$ & Sidra \\
\hline
\end{tabular}

Todos os coeficientes foram calculados pela razão simples entre as variáveis brutas apresentadas na segunda coluna do Quadro 1. Entretanto, mesmo agregando os dados de dois anos e no nível das microrregiões, observaram-se enormes variações aleatórias nos indicado- 
res, aconselhando o uso de algum método de atenuação. Por isso, a esses coeficientes brutos foi depois aplicado o método bayesiano empírico, que é um estimador que permite recalcular as taxas a um valor condizente com a população (nesse caso, óbitos) observada. De acordo com Câmara e outros (2004), o bayesiano empírico parte do pressuposto de que a taxa $t$ é uma variável aleatória, que tem uma média $m$ e uma variância $s$. Então, para calculá-lo, combinam-se a taxa observada $t_{i}$, a média $\left(m_{i}\right)$ e o peso de confiança $\left(w_{i}\right)$ do indicador para cada microrregião, conforme descrito na equação 2.1:

\section{$\Theta_{i}=w_{i} t_{i}+\left(1-w_{i} \mu_{i}\right)$ [equação 2.1]}

$\bigcirc$ fator $w_{i}$ é dado por:

$$
w_{i}=\frac{\sigma_{1}^{2}}{\sigma_{1}^{2}+u_{i} / n_{i}}
$$

Onde $u_{i}$ é a média aritmética global e $n_{i}$, a população da área i.

Portanto, por esse método, estimaram-se os coeficientes das microrregiões pela aplicação de uma média ponderada cujo fator $\left(w_{i}\right)$, ou peso de confiança, varia conforme o tamanho da população em estudo, sendo sempre menor quanto menor ela for, e vice-versa.

Da mesma forma que os coeficientes, a RMSUS foi calculada tendo em vista a relação entre o número de médicos e a população absoluta no mesmo lugar e período. Mas, nesse caso, como foi calculado em relação à população absoluta, não foram encontradas oscilações estatísticas que justificassem reestimar o indicador. As especialidades médicas selecionadas (médico da estratégia saúde da família, médico de família e comunidade, clínico geral e pediatra) foram definidas tendo em vista a importância de sua presença no acompanhamento do prénatal, do recém-nascido e da mãe.

Finalmente, o IPS, que é um indicador composto de privação, foi construído a partir da seleção de três indicadores estratégicos relativos ao território e capazes de determinar situações contextuais e sociais que influenciam a saúde materna e infantil, a saber: índice de analfabetismo da mulher em idade reprodutiva, índice de domicilios particulares permanentes sem instalação sanitária em seu interior e índice de domicílios com renda de até meio salário-mínimo. As variáveis foram selecionadas tendo em vista as dimensões transversais elementares da política de prevenção e promoção da saúde infantil por meio de ações na educação, na moradia e na distribuição da renda (Faria; Santana, 2015; Faria, 2013; Goldani et al., 2001). A partir das orientações originalmente oferecidas por Cartairs e Morris (1991), padronizamos essas variáveis pelo método $z$-score (equação 2.2) de modo que cada uma tivesse a mesma influência no resultado final do índice. Em síntese, o IPS representa a soma dessas variáveis padronizadas.

$$
Z_{\mathrm{s}}=\frac{x_{i}-\mu}{\sigma}[\text { equação 2.2] }
$$

Onde: $Z_{i}$ é a variável normalizada, $x_{i}$ é o escore bruto da variável, $\mu$ é a média aritmética e o é o desvio padrão amostral. 


\section{(b) Análise e representações espaciais}

Para as análises, aplicamos técnicas exploratórias de dados espaciais. Uma primeira aproximação experimental foi feita pela verificação da distribuição da frequência das variáveis (em histograma), tendência central (média aritmética) e dispersão (desvio padrão, coeficiente de variação). Antes, porém, foi necessário definir o número de classes ( $k$ ) a analisar. É consenso entre especialistas que o número de elementos $(n)$ da amostra deve ser relacionado ao número de classes a estimar (Ferreira, 2014). Considerando que são 558 microrregiões, por exemplo, teríamos um número arredondado de doze classes. Para efeitos, entretanto, esse método não seria pertinente aqui, pois agruparia numa mesma classe valores muito próximos e dificilmente reconhecíveis na escala do Brasil. Por isso, consideramos as variações da média, do mínimo e do máximo para todos os indicadores e acrescentamos mais dois cortes intermediários entre os valores acima e abaixo da média. Assim, tivemos cinco classes definidas e cuja representação gráfica pôde ser mais bem percebida e compreendida.

A representação gráfica ou cartográfica das classes, por sua vez, foi feita com recurso ao método de quebras naturais (natural breaks ou Jenks). Assim foi definido para evitar a existência de classes vazias e também porque este método permite refletir a variabilidade real dos valores da variável ou indicador (Ferreira, 2014).

Todas as análises ora descritas foram feitas em Sistema de Informação Geográfica (SIG). Para isso, utilizou-se o Programa ArcGis, da empresa Esri, disponível no laboratório de geoprocessamento do Departamento de Geociências da Universidade Federal de Santa Maria. A base cartográfica, na escala 1:200.000 e em Sistema de Projeção Sirgas 2000, foi obtida na plataforma digital do IBGE.

Finalmente, representamos os indicadores analisados em cartografia temática, por meio da qual se discutiram os resultados apresentados.

\section{Apresentação dos resultados}

\section{(a) Coeficiente de mortalidade infantil (CMI)}

O Brasil registrou 5.809.816 nascidos vivos e 78.089 óbitos infantis nos anos de 2012 e 2013. Isso representou um coeficiente de mortalidade infantil (CMI) na ordem de 13,4\%o. Quanto aos valores estimados pelo método bayesiano empírico, entretanto, a média do CMI do Brasil nesse período foi de 13,9\%. A Figura 1 mostra os resultados estimados no nível das microrregiões e com intervalos de coorte intencionalmente realizados em 13,9\%o (a média) e 15,7\% (o indicador pactuado no âmbito dos ODM para 2015) (WHO, 2005).

Os valores máximo e mínimo (8,1\%o e 34,0\%o, respectivamente) dão conta das ainda persistentes e enormes disparidades territoriais na distribuição desse indicador. Efetivamente, microrregiões com CMI acima de 18,5\% estão presentes apenas nos estados do Nordeste, do Norte e do Centro-Oeste. Ao contrário, com exceção da microrregião de Campo Grande-MS, as microrregiões com CMI abaixo de um dígito $(9,9 \%$ ) estão apenas nos estados das regiões Sudeste e Sul do país. Sem embargo, há duas realidades facilmente identificadas no mapa, por um Norte-Nordeste com piores resultados, um Sul-Sudeste com melhores resultados e sendo o Centro-Oeste uma área híbrida ou de transição entre CMI baixos e altos. 
Figura 1 - Coeficiente de mortalidade infantil (CMI) estimado por microrregião brasileira (2012-2013)
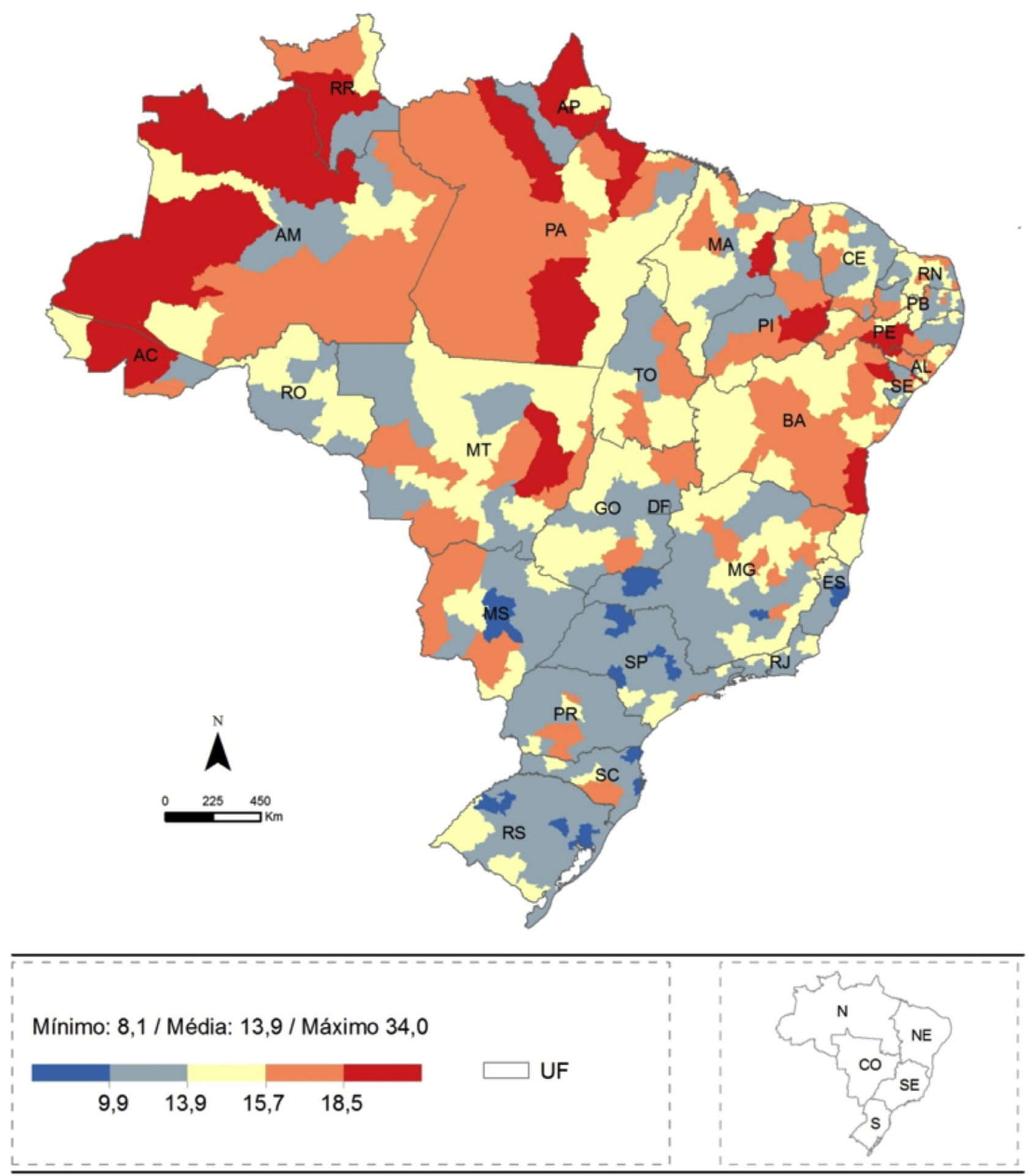

É também mais homogênea a distribuição espacial do indicador nos estados do Sudeste-Sul, embora também aí se observem CMI acima da média do país. Isso ocorre no interior de Santa Catarina e do Paraná (Curitibanos-SC e Guarapuava-PR, por exemplo), no sul do Rio Grande do Sul (municípios pampianos de fronteira), no litoral de São Paulo 
(Santos e Registro, por exemplo) e no norte de Minas Gerais (vale do Jequitinhonha e Zona da Mata Mineira). Já os estados do Nordeste-Norte apresentam uma distribuição bem mais heterogênea. Difícil inclusive mencionar especificidades. Senão, em relação ao Nordeste, são menos elevados os CMI nas microrregiões da Zona da Mata e mais elevados no interior (no sertão). Mas isso não é uma regra. Na Bahia, por exemplo, são observados elevados CMI em Ilhéus e Itabuna, que são litorâneas. No Norte, as variações são ainda maiores que no Nordeste. Há concentração espacial significativa de elevados CMI nas microrregiões localizadas entre o Acre, o oeste e o norte do estado do Amazonas e o estado de Roraima, de um lado, e do centro-sul do Pará até o estado do Amapá. Tocantins e Rondônia parecem ser os estados com os melhores CMI da região Norte.

\section{(b) Coeficientes de óbitos infantis relacionados a prematuridade (Coip), baixo peso no nascimento (Coibp), doenças infecciosas e parasitárias (Coidip) e adolescência da mãe (Coima)}

A Figura 2 mostra os resultados proporcionais estimados de óbitos infantis conforme quatro tipologias selecionadas: idade da mãe (canto superior esquerdo), algumas doenças infecciosas (canto superior direito), baixo peso (canto inferior esquerdo) e prematuridade (canto inferior direito). Consideremos cada tipologia separadamente.

São significativamente mais elevados os valores proporcionais de óbitos infantis entre os nascidos vivos de mães adolescentes de 10 a 19 anos nas microrregiões do Norte e do Nordeste (Coima). Por outro lado, são bem mais baixos esses valores nas microrregiões dos estados do Sudeste e do Sul e entre o Distrito Federal e Goiânia, no Centro - Oeste. A média brasileira é alta (20,6\%) e revela o impacto da gravidez na adolescência no cômputo final da mortalidade infantil. Mas, em boa parte do Nordeste e do Norte, mais de 30\% dos óbitos infantis são relativos a gravidez precoce. Devem-se destacar os estados do Amazonas e do Pará, de um lado, e São Paulo e Minas Gerais, de outro, que apresentaram, respectivamente, os maiores e menores valores proporcionais de óbitos infantis de mães adolescentes.

Os valores proporcionais estimados de óbitos infantis relacionados a algumas doenças infecciosas (Coidi) apresentaram uma distribuição espacial relativamente parecida com os valores proporcionais dos Coima. Portanto, são mais elevados os valores das microrregiões do Nordeste e do Norte e mais baixos os das localizadas no Sudeste e no Sul (novamente incluindo o Distrito Federal).

Os valores mais elevados de Coidi, com intervalo de coorte entre 7,4\% e 11,3\%, com exceção de uma microrregião do Mato Grosso do Sul (Dourados), são encontrados apenas nos estados do Nordeste e do Norte, com destaque para Pernambuco e Amazonas. Portanto, há um impacto significativamente elevado das doenças infecciosas no total de óbitos infantis nesses locais. 
Figura 2 - Coeficientes de óbito infantil por tipologias selecionadas: idade da mãe (Coima); doenças infecciosas (Coidi); baixo peso no nascimento (Coibp) e prematuridade (Coip)

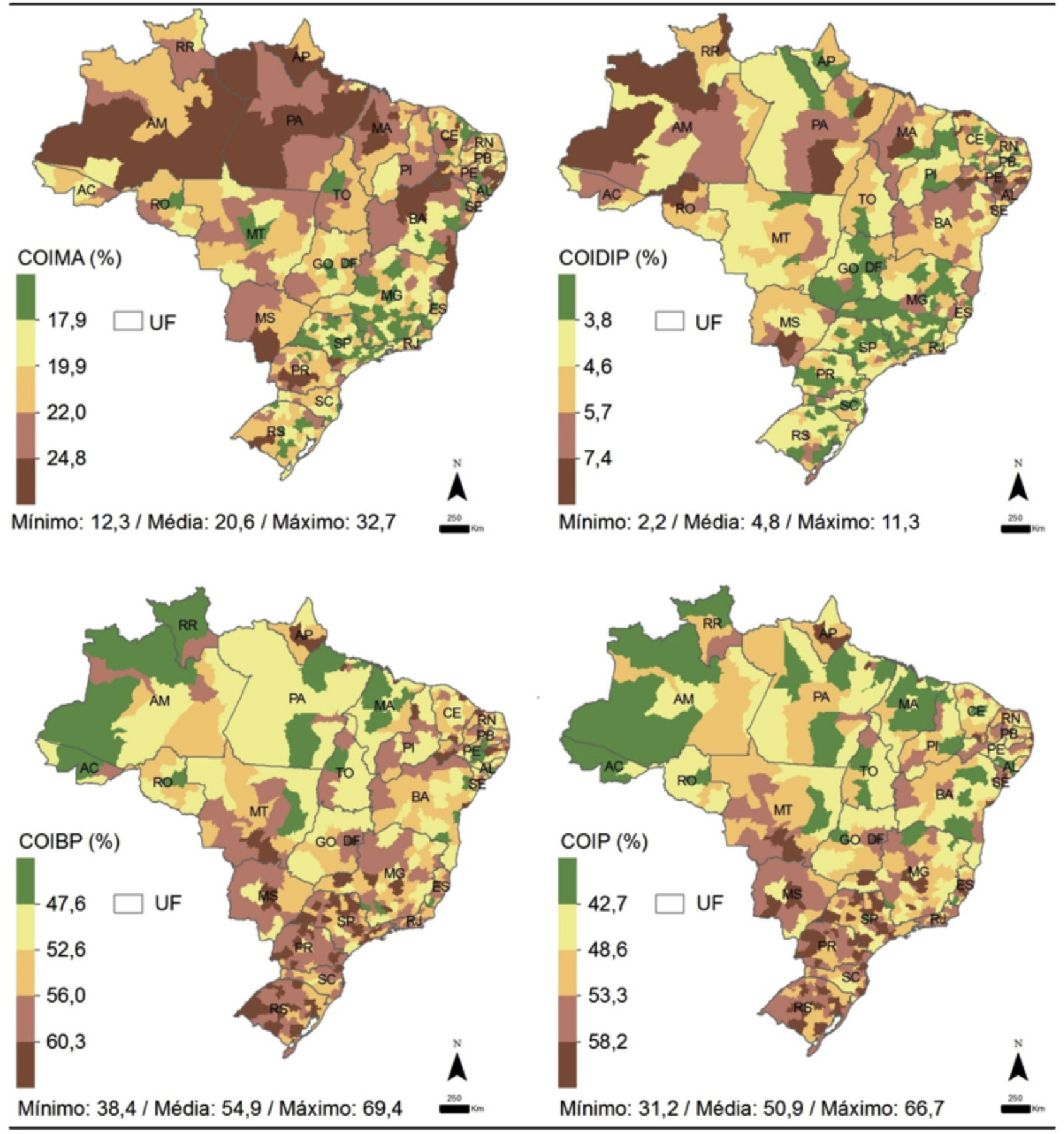

A distribuição espacial dos valores proporcionais de óbitos infantis relacionados aos nascidos vivos com baixo peso (Coibp) e nascidos vivos prematuros (Coip) é quase oposta aos valores proporcionais dos Coima e Coidi. Nesse caso, ao valores mais elevados são encontrados nas microrregiões dos estados do Sudeste e do Sul e de grande parte do Centro-Oeste, e os mais baixos, nas microrregiões do Nordeste e do Norte. No primeiro grupo, predominam os valores proporcionais de Coibp e Coip acima de 50\% (cores marrom claro e escuro) e, no segundo, predominam valores proporcionais abaixo desse valor (cores amarelo e verde). 
Um fator explicativo dessa distribuição, por exemplo, é que apenas três (Coibp) e cinco (Coip) microrregiões, em verde, estão nos estados do Sudeste e Sul e algumas dezenas nos estados do Nordeste e do Norte. Por outro lado, os valores proporcionais mais elevados, representados pelo intervalo de coorte acima de 60,3\% e 58,2\% (respectivamente, Coibp e Coip), em marrom escuro, são restritos a algumas microrregiões dos estados do Nordeste e do Norte e abundantes nos estados do Sudeste e do Sul e de grande parte do Centro-Oeste.

\section{(c) Atenção médica e pré-natal}

A atenção médica à saúde do SUS, responsável pelo acompanhamento da gestante e do bebê em seus primeiros meses de vida, é representada principalmente pelas especialidades da estratégia saúde da família, médico de familia e comunidade, clínico geral e pediatra. Nessas especialidades e considerando os dados agregados do segundo do ano de 2012, o Brasil apresentou uma média de 0,5 médico por mil habitantes. Mas esse número varia de 0,1 (predominante nas microrregiões do Nordeste e do Norte) a 1,3 (predominante no Sudeste e no Sul). Portanto, os índices mais elevados de cobertura médica à saúde do SUS são encontrados nas microrregiões do Sudeste e do Sul e em boa parte do Centro-Oeste, ao passo que os índices menos elevados predominam sobretudo nas microrregiões nordestinas e do Norte, envolvendo também extensas áreas do norte de Minas Gerais e do Mato Grosso (Figura 3).

São relativamente parecidas as distribuições espaciais da cobertura médica e do uso do pré-natal no Brasil. Em grande parte das microrregiões do Norte e do Nordeste brasileiros, menos da metade das gestantes fizeram o acompanhamento ideal propugnado pelo Ministério da Saúde (Brasil, 2006), de sete ou mais consultas pré-natais.

Nas microrregiões do Sudeste e do Sul e, novamente, de boa parte do Centro-Oeste, predomina o Inv $\geq 7 \mathrm{CPN}$ acima 76,3\%, chegando ao máximo de 88,7\% dos nascidos vivos. Ao contrário, em grande parte do Nordeste e do Norte, o Inv $\geq 7 C P N$ não ultrapassa 41,3\%, chegando ao impressionante valor mínimo de $13,5 \%$ dos nascidos vivos.

\section{Figura 3 - Distribuição espacial do índice de médicos do SUS (especialidades selecionadas) por habitante (IMSUS) e índice de nascidos vivos que fizeram} sete ou mais consultas pré-natais (Inv $\geq 7 \mathrm{CPN}$ ) por microrregião brasileira

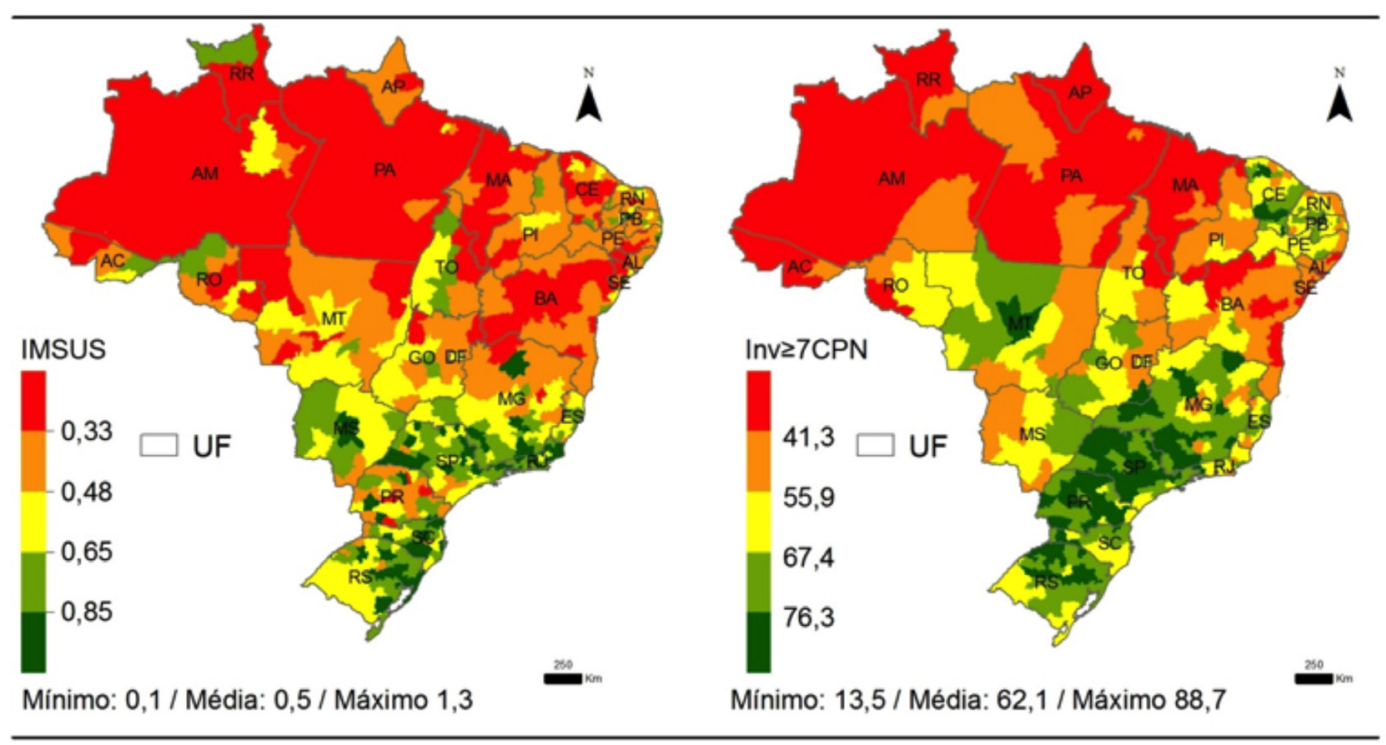




\section{(d) Índice de privação social (IPS)}

A privação social, medida nesse estudo com os indicadores de analfabetismo da mulher em idade reprodutiva, domicilios sem banheiro no seu interior e baixa renda por domicílio, é um indicador transversal e de contexto importante para entender a relação entre a produção do território brasileiro e a produção de condições favoráveis ou desfavoráveis ao desenvolvimento da vida. Infelizmente, ainda predominam no Brasil duas realidades, representadas por lugares menos (Sul, Sudeste e parte do Centro-Oeste) e mais carenciados (Nordeste e Norte). A mortalidade infantil desigual é ainda resultado dessa dupla realidade.

São facilmente reconhecíveis na Figura 4 microrregiões menos carenciadas e cujo IPS é negativo e microrregiões mais carenciadas, cujo IPS é positivo. Não há microrregiões em rosa (IPS acima de 4,2, chegando ao máximo de 7,7), por exemplo, nos estados do Sudeste, do Sul ou do Centro-Oeste. Elas predominam, aliás, em extensões muito parecidas com os elevados $\mathrm{CMI}$, no interior do Nordeste e entre o Acre e Amazonas.

Figura 4 - Distribuição espacial do índice de privação social (IPS) e índice de nascidos vivos de mães de 10 a 19 anos de idade (InvM10-19) por microrregião brasileira

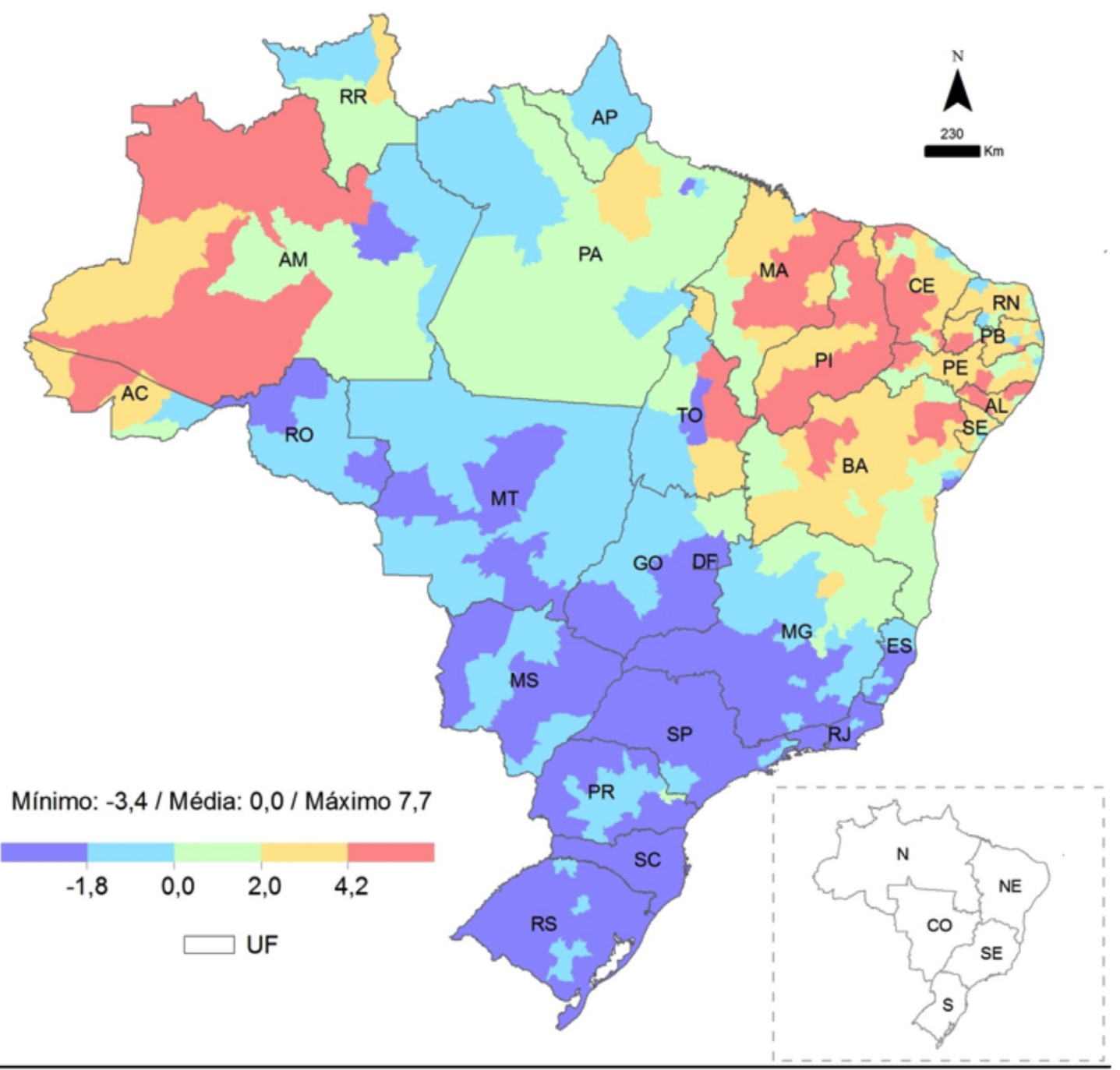


Especificamente no Nordeste, o IPS é ainda mais elevado nas microrregiões do Maranhão e do Piauí e no oeste do Ceará. E também ficam no interior, na área sertaneja desses estados, os lugares mais carenciados. Variações também são encontradas nos estados da região Sudeste. É o caso do norte de Minas Gerais, onde são encontrados IPS bem mais elevados. Índices mais elevados são também encontrados na região central do Paraná (ao redor de Guarapuava) e no Pampa Gaúcho. No Centro-Oeste, os IPS mais elevados são encontrados nos estados de Mato Grosso e Mato Grosso do Sul e no norte de Goiás.

\section{Discussão dos resultados}

Efetivamente, a mortalidade infantil é um problema do território. Suas variações espaciais refletem bem e talvez como nenhum outro indicador as desigualdades territoriais. Para os mais otimistas ou até mesmo os mais críticos, há razões para comemorar os avanços nas políticas de redução da mortalidade infantil no Brasil. Não sem razão e também não sem alguma ingenuidade. De fato, é preciso valorizar o feito, mas é também preciso relativizar os números, humanizá-los talvez (se é que isso é possível), afinal, foram mais de 78 mil óbitos de crianças que não puderam comemorar seu primeiro ano de vida entre os anos de 2012 e 2013.

Obviamente, não existe nenhum país do mundo com coeficiente zero de mortalidade infantil, mas são ainda enormes os desafios da redução do óbito infantil no Brasil, porque quase tudo aqui acaba exigindo esforços gigantescos (seja pela dimensão, pela enorme população ou pela própria história do país); além disso, também porque as respostas são geralmente lentas e descompassadas. Antecipe-se, não é possível uma política única de redução da mortalidade infantil para todo o país. Observe-se a Figura 1 para ver que o país apresenta diferentes níveis de desenvolvimento nesse indicador. E não são apenas níveis de desenvolvimento baixo e elevados, mas diferentes níveis de desenvolvimento no território. Por isso, as políticas de redução da mortalidade infantil no Brasil devem se adequar às necessidades do território, a seus perfis (Faria, 2013). Efetivamente, a média brasileira não deve ser critério de comparação ou valor tipo ideal para o desenvolvimento de políticas de saúde infantil. É a realidade do território que se deve levar em conta, pois qualquer equivalência que se queira estabelecer só pode ser feita em relação ao próprio território ou à própria realidade territorial. Em outras palavras, as microrregiões do Sudeste e do Sul e de parte do Centro-Oeste só podem ser discutidas em relação a si mesmas, e não às microrregiões do Nordeste ou do Norte, por exemplo.

Apresenta-se, pois, a necessidade de reconhecer os reais desafios a serem enfrentados pelo Brasil nas políticas de redução da mortalidade infantil nas próximas décadas. De um ponto de vista geral, não é preciso insistir na existência de um quadro de dificuldades enormes, talvez mais complexas que as da década de 1990. Com efeito, o Brasil vive hoje não só uma realidade de descompassos (Norte-Nordeste, de um lado, Centro-Sul, de outro), mas também uma encruzilhada. Senão, vejamos.

Os descompassos são facilmente identificados pelas profundas desigualdades territoriais em todos os indicadores analisados. Na verdade e com recurso à generalização, são facilmente reconhecíveis no Brasil duas realidades assim definidas: Norte e Nordeste mais carenciados, Sudeste e Sul, menos (porque não seria adequado falar em Sudeste e Sul desenvolvidos, por 
exemplo), o Centro-Oeste apresentando extensões menos carenciadas entre Brasília e Goiânia e outras mais carenciadas, nos estados de Mato Grosso e Mato Grosso do Sul. As variações do coeficiente de mortalidade infantil, assim como os fatores associados (atenção médica, acesso ao pré-natal, idade da mãe, doenças infecciosas, prematuridade e baixo peso no nascimento) refletem essas duas realidades.

Infelizmente, os CMI são ainda muito elevados nas microrregiões dos estados do Nordeste e do Norte, acima do que se podia esperar nesta segunda década do século XXI. $\bigcirc$ Brasil também ainda não resolveu o problema dos vazios assistenciais nesses estados e tampouco a precária atenção médica à gestante. Por isso, menos da metade das gestantes residentes nessas microrregiões tiveram acompanhamento regular recomendado de pelo menos sete consultas pré-natal. E esse problema, é bom que se diga, é elementar na política de redução da mortalidade infantil, relembra mesmo os discursos da política nacional da década de 1980. Como não podia deixar de ser, as microrregiões mais carenciadas do Norte e do Nordeste também apresentam valores proporcionais mais elevados de óbitos infantis nascidos de mães adolescentes (de 10 a 19 anos) e óbitos relativos a doenças infecciosas. Portanto, embora genérico, é um quadro que revela enorme atraso nas políticas de desenvolvimento territorial.

Realidade muito distinta é vivida em boa parte das microrregiões dos estados do Sudeste e do Sul do Brasil, envolvendo também parte do Centro-Oeste, sobretudo Goiás e Brasília. Nesses locais, são encontrados os menores CMI (com exceção do norte de Minas Gerais), e o problema assistencial perde importância, o acesso ao acompanhamento pré-natal é facilitado, a gestação na adolescência é significativamente reduzida e são menores os impactos das doenças infecciosas no óbito infantil. Portanto, são áreas menos carenciadas, com os menores CMI e melhores condições assistenciais. Mas aí os desafios são outros, sobretudo a prematuridade e o baixo peso no nascimento.

De fato, mais de 50\% dos óbitos infantis das microrregiões dos estados do Sudeste e do Sul e de parte do Centro-Oeste podem ser relacionados, direta ou indiretamente, com o baixo peso e a prematuridade. Embora não estejam ausentes, são menos relevantes os fatores relativos à idade e à escolaridade da mãe, a doenças infecciosas e à cobertura médico-assistencial. Naturalmente, isso faz com que, em termos proporcionais, a prematuridade e o baixo peso sejam mais importantes. Portanto, a política de redução da mortalidade infantil nesses locais deve refletir essa especificidade territorial.

Como dissemos, acreditamos que o Brasil se coloca hoje numa situação delicada ou de encruzilhada em termos de política de desenvolvimento territorial da saúde infantil. De fato, essa realidade ambígua já impõe o desafio da redução das desigualdades. Isso não é novidade na política nacional. Contudo, a complexidade aumenta nesse momento porque os estados do Sudeste, ou pelo menos a maior parte deles, exigem políticas adequadas de combate à prematuridade e baixo peso. É o caso exemplificativo da recém-criada Resolução n. 368 da Agência Nacional de Saúde (ANS, 2015), que visa informar sobre os partos cesarianos realizados na rede particular de saúde. Além de minimizar os interesses financeiros da medicina do parto, a Resolução pretende, obviamente, reduzir o número de operações cesarianas e aumentar os partos naturais, reconhecendo que o parto natural concorre para a redução da prematuridade (Cascaes et al., 2008). 
Sem embargo, dificilmente os CMI dos estados do Sudeste e do Sul chegarão aos níveis de 3\% e 5\% encontrados nos países desenvolvidos sem uma política sistemática de redução da prematuridade e baixo peso no nascimento. Todavia, isso exigirá ações bem mais setorizadas, especializadas e dirigidas. De fato, os fatores de determinação básicos, como as doenças infecciosas, idade da mãe, analfabetismo, por exemplo, continuarão a existir e deverão ser enfrentados. Mas as políticas de enfrentamento, nesse caso, são bem mais transversais e, portanto, não restritas ao setor saúde apenas. As políticas educacionais desenvolvidas nas décadas de 1980, por exemplo, foram fundamentais para a redução da mortalidade infantil. $\bigcirc$ mesmo se pode dizer das ações de melhoria do saneamento, da redução do trabalho infantil e da criação de dispositivos legais de proteção à gestante no trabalho, entre outros. Isso não ocorre ou pelo menos não ocorre na mesma profundidade com a prematuridade e o baixo peso, que são políticas mais diretamente ligadas à saúde como setor. Ou seja, os esforços agora dependem mais de fatores assistenciais e médicos, relativos às ações de prevenção e promoção. E essa realidade provavelmente se aprofundará nas próximas décadas, generalizando-se para todo o país.

Efetivamente, há três quadros possíveis que, acreditamos, se poderão confirmar nas próximas décadas no Brasil: (i) continuidade da redução do CMI no nível das áreas desenvolvidas do globo (o que significaria diminuir em pelo menos $70 \%$ o valor médio do coeficiente atual); (ii) manutenção dos CMI atuais encontrados nos estados mais desenvolvidos e redução dos $\mathrm{CMI}$ atualmente elevados nos estados menos desenvolvidos (influenciados pela melhoria nos indicadores sociais básicos, os estados do Nordeste e do Norte apresentariam avanços, mas a dificuldade de implementar ações especializadas deixaria praticamente estacionado o CMI dos demais estados); (iii) retrocesso nas políticas de redução da mortalidade infantil em praticamente todo o país (num quadro de enorme crise social, política e econômica, os CMI não só não diminuiriam, como poderiam aumentar).

Evidentemente, tais especulações, não sem fundamento, podem simplesmente não se confirmar, e pode-se um quadro ainda mais complexo ou variável. $\bigcirc$ aprofundamento das desigualdades regionais pode, por exemplo, produzir um cenário em que as três situações conviverão. Interessa, entretanto, que, a partir das perspectivas atuais, se desenvolvam novas políticas, adequadas aos diferentes perfis do território brasileiro.

\section{Considerações finais}

É ainda longo o caminho a percorrer nas políticas de redução da mortalidade infantil no Brasil. Apresentam-se agora novos desafios (redução da prematuridade e do baixo peso), num quadro de não superação de desafios anteriores pactuados (redução do analfabetismo materno, diminuição da gravidez precoce e acesso aos serviços de saúde, entre outros).

Nas áreas menos carenciadas dos estados do Sudeste e do Sul e de parte do Centro-Oeste, o coeficiente de mortalidade infantil é baixo, mas ainda não suficiente para que se possa considerar socialmente adequado. É baixo em comparação com os estados do Nordeste e do Norte, sobretudo no interior desses estados, mas é ainda suficientemente alto para permitir uma redução de pelo menos 70\%. De fato, a maior parte dessas microrregiões menos carenciadas do Brasil ainda tem um $\mathrm{CMI}$ com dois dígitos, entre 10\% e 13,9\%. E, não descredenciando os avanços alcançados com grande esforço político e social, há ainda muito a se fazer para reduzir esses valores. 
Em situação mais delicada encontram-se as microrregiões dos estados do Nordeste e do Norte e de parte do Centro-Oeste. Ali, apresentam-se ainda os desafios elementares de reduzir um valor global acima de 15,7\%o, com locais apresentando valores acima de 20\%o. Considerando a atual realidade brasileira e mundial, não é preciso insistir no absurdo de tal coeficiente neste momento, sem deixar de mencionar problemas sérios relativos a doenças infecciosas e a complicações no parto e no puerpério, como o que vêm experimentando alguns estados do Nordeste com a encefalopatia, possivelmente ligada à epidemia do vírus zika. De fato, os eventos epidêmicos da dengue, associados às recentes importações dos vírus zika e chikungunya, todos transmitidos pelo mesmo vetor, são extremante preocupantes nos estados do Norte e do Nordeste e com grande impacto nos indicadores de saúde materna e infantil. Não é sem razão que o coeficiente de óbitos infantis relativos a doenças infecciosas e parasitárias (Coidp) foi bem mais alto nesses lugares, principalmente nas microrregiões do extremo Norte do país, e essas questões são ainda amplificadas pela manutenção da violência, do trabalho infantil, da pobreza e do trabalho manual forçado em áreas de agricultura e mineração, entre outras. A falta de acesso a serviços (médicos, por exemplo) e a bens (moradia e renda) é o principal problema a ser combatido nesses locais (devendo-se incluir o norte mineiro, parte do litoral paulista, a área central do Paraná, próxima a Guarapuava, e o Pampa gaúcho, entre outros).

Portanto, reconhecendo-se esses desafios novos e velhos, o Brasil pode criar políticas condizentes com a realidade territorial, evitando os discursos demasiadamente generalizantes sobre os avanços sociais recentes das ações de vigilância e controle da mortalidade infantil. $\bigcirc$ problema do controle ainda não foi resolvido no país. E isso é elementar, ou seja, não deixar morrer. Por isso, é fundamental implementar políticas territoriais integradas capazes de tornar mais resolutivas ações que garantam a sobrevida do bebê. Entretanto, embora elementar, não é suficiente não deixar morrer. Não adianta sobreviver e viver pouco ou mal. É preciso oferecer à criança qualidade de vida em toda a sua trajetória até a idade escolar, mas sobretudo e estrategicamente em seu primeiro ano de vida.

\section{Referências}

ANS. ASSOCIAÇÃO NACIONAL DE SAÚDE SUPLEMENTAR. Resolução Normativa n. 368, de 6 de janeiro de 2015. Dispõe sobre o direito de acesso à informação das beneficiárias aos percentuais de cirurgias cesáreas e de partos normais, por operadora, por estabelecimento de saúde e por médico e sobre a utilização do partograma, do cartão da gestante e da carta de informação à gestante no âmbito da saúde suplementar, 2015. Disponível em: http://www.ahseb.com.br/wp-content/uploads/2015/05/RN_N\%C2\%BA368.pdf. Acesso em: 21 maio 2015.

BRASIL. Ministério da Saúde. Consolidação do Sistema de Informações sobre Nascidos Vivos - 2011. Brasília: Ministério da Saúde/Secretaria de Vigilância Epidemiológica, 2012.

Pré-natal e puerpério: atenção qualificada e humanizada - manual técnico. Brasília: Ministério da Saúde, 2006. 
CÂMARA, G. et al. Análise espacial em áreas. In: DRUCK, S. et al. (Org.). Análise espacial de dados geográficos. Brasília: Embrapa, 2004. p. 155-205.

CAMPBELL, M.; APPARICIO, P.; DAY, P. Geographic analysis of infant mortality in New Zealand, 1995-2008: an ethnicity perspective. Australian and New Zealand Journal of Public Health, Sydney, v. 38, n. 3, p. 221-226, 2015.

CARTAIRS, V.; MORRIS, R. Deprivation and health in Scotland. Aberdeen: Aberdeen University Press, 1991.

CARVALHO, R. A. S. et al. Desigualdades em saúde: condições de vida e mortalidade infantil na região Nordeste do Brasil. Revista de Saúde Pública, Rio de Janeiro, v. 49, n. 5, p. 1-9, 2015.

CASCAES, A. M. et al. Prematuridade e fatores associados no estado de Santa Catarina, Brasil, no ano de 2005: análise dos dados do Sistema de Informações sobre Nascidos Vivos. Cadernos de Saúde Pública, Rio de Janeiro, v. 24, n. 4, p. 1024-1032, 2008.

COSTA, M. N. et al. Mortalidade infantil e condições de vida: a reprodução das desigualdades sociais em saúde na década de 90. Cadernos de Saúde Pública, Rio de Janeiro, v. 17 , n. 3, p. 555-567, 2001.

FARIA, R. Geografia da mortalidade materna e infantil no contexto das desigualdades regionais no Brasil. In: Congresso da Associação Portuguesa para o Desenvolvimento Regional, 19., 2013, Universidade do Minho. Anais... Braga: APDR, 2013. p. 725-737.

; SANTANA, P. Regional inequalities in infant mortality and primary health care in the State of Minas Gerais, Brazil. Space Populations and Societies, Paris, v. 2-3, p. 1-14, 2015.

FERREIRA, M. C. Iniciação à análise espacial: teoria, técnicas e exemplos para o geoprocessamento. São Paulo: Unesp, 2014.

GOLDANI, M. Z. et al. Infant mortality rates according to socioeconomic status in a Brazilian city. Revista de Saúde Pública, São Paulo, v. 35, n. 3, p. 256-261, 2001.

GUIMARÃES, R. Saúde: fundamentos de geografia humana. São Paulo: Editora Unesp Digital, 2015.

IBGE. INSTITUTO BRASILEIRO DE GEOGRAFIA E ESTATÍSTICA. Brasil em síntese: população. Disponível em: http://www.ibge.gov.br. Acesso em: 20 maio 2015.

Evolução e perspectivas da mortalidade infantil no Brasil. Rio de Janeiro: IBGE, 1999.

IPEA. INSTITUTO DE PESQUISA ECONÔMICA APLICADA. Objetivos de desenvolvimento do milênio. Brasilia: Ipea, 2014. Relatório Nacional de Acompanhamento.

NOLTE, E.; MCKEE, M. Does healthcare save lives? Avoidable mortality revisited. London: The Nuffield Trust, 2004.

NORMAN, P. et al. Geographical trends in infant mortality: England and Wales, 1970-2006. Health Statistic Quarterly, United Kingdom, v. 40, p. 18-29, 2008. 
SANTOS, M. O espaço do cidadão. São Paulo: Nobel, 1987.

SZWARCWALD, C. L.; ANDRADE, C. T.; BASTOS, F. I. Income inequality, residential poverty clustering and infant mortality: a study in Rio de Janeiro, Brazil. Social Science $\mathcal{E}$ Medicine, London, v. 55, n. 12, p. 2083-2092, 2002.

UNICEF. UNITED NATIONS CHILDREN'S FUND. Report 2014: levels and trends in child mortality. New York: United Nations Inter-agency Group for Child Mortality Estimation, 2014. Disponível em: http://www.unicef.org/media/files >. Acesso em: 15 set. 2015.

VICTORA, C. G.; BARROS, F. C. Infant mortality due to perinatal causes in Brazil: trends, regional patterns and possible interventions. São Paulo Medical Journal, v. 119, p. 33-42, 2001.

WHO. WORLD HEALTH ORGANIZATION. Health and the Millennium Development Goals. Geneva: WHO, 2005.

PREFEITURA MUNICIPAL DE PELOTAS. Plano local de habitação de interesse social. Pelotas, 2012.

SÁ BRITTO, N. D. Industrialização e desindustrialização do espaço urbano em Pelotas-RS. Dissertação (Mestrado em Geografia) - Instituto de Ciências Humanas e da Informação, Universidade Federal do Rio Grande, Rio Grande, 2011.

SALGUEIRO, T. B. Cidade Pós-moderna: espaço fragmentado. Revista território, v. III, n. 4, jan./jun. 1998.

SHIMBO, L. Z. Habitação social, habitação de mercado: a confluência entre Estado, empresas construtoras e capital financeiro. Tese (Doutorado em Arquitetura) - Escola de Engenharia de São Carlos, Universidade de São Paulo, São Carlos, 2010.

SOARES, P. R. R. Produção imobiliária e crescimento urbano em cidades médias: Pelotas e Rio Grande-RS. In: SILVEIRA, R. L.; PEREIRA, P. X.; UEDA, V. (Org.). Dinâmica imobiliária e reestruturação urbana na América Latina. Santa Cruz do Sul: Edunisc, 2006. p. 159-192.

Del proyecto urbano a la producción del espacio: morfología urbana de la ciudad de Pelotas, Brasil (1812-2000). Tese (Doutorado em Geografia) - Departamento de Geografía Humana, Universidad de Barcelona, Barcelona, 2002.

SOJA, E. W. Geografias pós-modernas: a reafirmação do espaço na teoria social crítica. Rio de Janeiro: Jorge Zahar, 1993.

VASCONCELOS, P. A. Contribuições para o debate sobre processos e formas socioespaciais nas cidades. In: VASCONCELOS, P. A.; CORREA, R. L.; PINTAUDI, M. (Org.). A cidade contemporânea: segregação espacial. São Paulo: Contexto, 2013. p. 17-37.

VIEIRA, S. G. A cidade fragmentada: o planejamento e a segregação social do espaço urbano em Pelotas. Pelotas: Editora da UFPEL, 2005. 